\title{
Interval-Valued Fuzzy Cosets
}

\author{
Keon Chang Lee*, Kul Hur** and Pyung Ki Lim***† \\ *Department of Computer Science, Dongshin University \\ ${ }^{* *, * * *}$ Division of Mathematics and Informational Statistics, and Nanoscale Science and Technology Institute, \\ Wonkwang University
}

\begin{abstract}
First, we prove a number of results about interval-valued fuzzy groups involving the notions of interval-valued fuzzy cosets and interval-valued fuzzy normal subgroups which are analogs of important results from group theory. Also, we introduce analogs of some group-theoretic concepts such as characteristic subgroup, normalizer and abelian groups. Secondly, we prove that if $A$ is an interval-valued fuzzy subgroup of a group $G$ such that the index of $A$ is the smallest prime dividing the order of $G$, then $A$ is an interval-valued fuzzy normal subgroup. Finally, we show that there is a one-to-one correspondence the interval-valued fuzzy cosets of an interval-valued fuzzy subgroup $A$ of a group $G$ and the cosets of a certain subgroup $H$ of $G$.
\end{abstract}

Key Words: interval-valued fuzzy normal subgroup, interval-valued fuzzy coset, interval-valued fuzzy characteristic fuzzy subgroup, normalizer, abelian group.

\section{Introduction}

The concept of a fuzzy set was introduced by Zadeh[9], and in 1965, he[10] introduced the notion of intervalvalued fuzzy set as a generalization of fuzzy sets. After that time, Mondal and Samanta[8], and choi et al.[3] applied it to topology. Also, several researchers [1,2, 4-7] applied one to algebra.

The present paper is a sequel to [4]. We obtain a number of further analogs of the properties of groups, thereby enriching the theory of interval-valued fuzzy groups and, in particular, corroborating the concept of interval-valued fuzzy normal subgroups and interval-valued fuzzy cosets introduced in $[4,5]$. Moreover, we obtain an analog of the

접수일자 : 2012년 5월 23일

심사(수정)일자 : 2012년 10월 15일

게재확정일자 : 2012년 10월 16일

$\dagger$ 교신저자 following standard result from group theory that if $\theta$ is an automorphism of a group $G$ which leaves invariant some normal subgroup $N$, then $\theta$ induces an automorphism of the quotient group $G / N$.

Some variations of this result are also considered, for which we obtain analogs for interval-valued fuzzy groups. Also we show that there is a natural one-to-one correspondence between the interval-valued fuzzy cosets of an interval-valued fuzzy subgroup $A$ of a group $G$ and the cosets of a subgroup $G_{A}$ of $G$ defined by $G_{A}=\{g \in G$ : $A(g)=A(e)\}$, where $e$ denotes, as usual, the identity element of the group $G$. Our analysis illustrates that the subgroup $G_{A}$ defined above plays a significant role in investigating the structure of the corresponding interval-valued fuzzy subgroup.

\section{Preliminaries}

In this section, we list some basic concepts and well- 
known results which are needed in the later sections.

Let $D(I)$ be the set of all closed subintervals of the unit interval $I=[0,1]$. The elements of $D(I)$ are generally denoted by capital letters $M, N, \cdots$, and note that $M=\left[M^{L}, M^{U}\right]$, where $M^{L}$ and $M^{U}$ are the lower and the upper end points respectively. Especially, we denoted, $\mathbf{0}=[0,0], \mathbf{1}=[1,1]$, and $\boldsymbol{a}=[a, a]$ for every $a \in(0,1)$. We also note that

(i) $(\forall M, N \in D(I))\left(M=N \Leftrightarrow M^{L}=N^{L}, M^{U}=\right.$ $\left.N^{U}\right)$

(ii) $(\forall M, N \in D(I))\left(M \leq N \Leftrightarrow M^{L} \leq N^{L}, M^{U} \leq\right.$ $\left.N^{U}\right)$.

For every $M \in D(I)$, the complement of $M$, denoted by $M^{c}$, is defined by $M^{c}=1-M=\left[1-M^{U}, 1-M^{L}\right]$ (See [8]).

Definition 2.1 [8, 10]. A mapping $A: X \rightarrow D(I)$ is called an interval-valued fuzzy set (in short, IVS) in $X$, denoted by $A=\left[A^{L}, A^{U}\right]$, if $A^{L}, A^{U} \in I^{X}$ such that $A^{L} \leq A^{U}$, i.e., $A^{L}(x) \leq A^{U}(x)$ for each $x \in X$, where $A^{L}(x)$ [resp. $\left.A^{U}(x)\right]$ is called the lower[resp. upper] end point of $x$ to $A$. For any $[a, b] \in D(I)$, the interval-valued fuzzy set $A$ in $X$ defined by $A(x)=\left[A^{L}(x), A^{U}(x)\right]=[a, b]$ for each $x \in X$ is denoted by $\widetilde{[a, b}]$ and if $a=b$, then the IVS $\widetilde{[a, b]}$ is denoted by simply $\widetilde{a}$. In particular, $\widetilde{0}$ and $\widetilde{1}$ denote the interval-valued fuzzy empty set and the interval-valued fuzzy whole set in $X$, respectively.

We will denote the set of all IVSs in $X$ as $D(I)^{X}$. It is clear that set $A=\left[A^{L}, A^{U}\right] \in D(I)^{X}$ for each $A \in I^{X}$.

Definition 2.2 [8]. Let $A, B \in D(I)^{X}$ and let $\left\{A_{\alpha}\right\}_{\alpha \in \Gamma} \subset$ $D(I)^{X}$. Then

(i) $A \subset B$ iff $A^{L} \leq B^{L}$ and $A^{U} \leq B^{U}$.

(ii) $A=B$ iff $A \subset B$ and $B \subset A$.

(iii) $A^{c}=\left[1-A^{U}, 1-A^{L}\right]$.

(iv) $A \cup B=\left[A^{L} \vee B^{L}, A^{U} \vee B^{U}\right]$.

(iv) $^{\prime} \bigcup_{\alpha \in \Gamma} A_{\alpha}=\left[\bigvee_{\alpha \in \Gamma} A_{\alpha}^{L}, \bigvee_{\alpha \in \Gamma} A_{\alpha}^{U}\right]$.

(v) $A \cap B=\left[A^{L} \wedge B^{L}, A^{U} \wedge B^{U}\right]$.

$(\mathrm{v})^{\prime} \bigcap_{\alpha \in \Gamma} A_{\alpha}=\left[\bigwedge_{\alpha \in \Gamma} A_{\alpha}^{L}, \bigwedge_{\alpha \in \Gamma} A_{\alpha}^{U}\right]$
Result2.A $\left[8\right.$, Theorem1]. Let $A, B, C \in D(I)^{X}$ and let $\left\{A_{\alpha}\right\}_{\alpha \in \Gamma} \subset D(I)^{X}$. Then
(a) $\tilde{\mathbf{0}} \subset A \subset \tilde{\mathbf{1}}$.
(b) $A \cup B=B \cup A, A \cap B=B \cap A$.
(c) $A \cup(B \cup C)=(A \cup B) \cup C, A \cap(B \cap C)=$ $(A \cap B) \cap C$.

(d) $A, B \subset A \cup B, A \cap B \subset A, B$.

(e) $A \cap\left(\bigcup_{\alpha \in \Gamma} A_{\alpha}\right)=\bigcup_{\alpha \in \Gamma}\left(A \cap A_{\alpha}\right)$.

(f) $A \cup\left(\bigcap_{\alpha \in \Gamma} A_{\alpha}\right)=\bigcap_{\alpha \in \Gamma}\left(A \cup A_{\alpha}\right)$.

(g) $(\tilde{\mathbf{0}})^{c}=\tilde{\mathbf{1}},(\tilde{\mathbf{1}})^{c}=\tilde{\mathbf{0}}$.

(h) $\left(A^{c}\right)^{c}=A$.

(i) $\left(\bigcup_{\alpha \in \Gamma} A_{\alpha}\right)^{c}=\bigcap_{\alpha \in \Gamma} A_{\alpha}^{c},\left(\bigcap_{\alpha \in \Gamma} A_{\alpha}\right)^{c}=\bigcup_{\alpha \in \Gamma} A_{\alpha}^{c}$.

Definition 2.3 [8]. Let $f: X \rightarrow Y$ be a mapping, let $A=\left[A^{L}, A^{U}\right] \in D(I)^{X}$ and let $B=\left[B^{L}, B^{U}\right] \in D(I)^{Y}$. Then

(a) the image of $A$ under $f$, denoted by $f(A)$, is an IVS in $Y$ defined as follows: For each $y \in Y$,

$$
f\left(A^{L}\right)(y)= \begin{cases}\bigvee_{y=f(x)} A^{L}(x), & \text { if } f^{-1}(y) \neq \emptyset ; \\ 0, & \text { otherwise. }\end{cases}
$$

and

$$
f\left(A^{U}\right)(y)= \begin{cases}\bigvee_{y=f(x)} A^{U}(x), & \text { if } f^{-1}(y) \neq \emptyset ; \\ 0, & \text { otherwise. }\end{cases}
$$

(b) the preimage of $B$ under $f$, denoted by $f^{-1}(B)$, is an IVS in $Y$ defined as follows: For each $y \in Y$,

$$
f^{-1}\left(B^{L}\right)(y)=\left(B^{L} \circ f\right)(x)=B^{L}(f(x))
$$

and

$$
f^{-1}\left(B^{U}\right)(y)=\left(B^{U} \circ f\right)(x)=B^{U}(f(x)) .
$$

It can be easily seen that $f(A)=\left[f\left(A^{L}\right), f\left(A^{U}\right)\right]$ and $f^{-1}(B)=\left[f^{-1}\left(B^{L}\right), f^{-1}\left(B^{U}\right)\right]$.

Result 2.B [8, Theorem 2]. Let $f: X \rightarrow Y$ be a mapping and $g: Y \rightarrow Z$ be a mapping. Then

(a) $f^{-1}\left(B^{c}\right)=\left(f^{-1}(B)\right)^{c}, \forall B \in D(I)^{Y}$. 
(b) $[f(A)]^{c} \subset f\left(A^{c}\right), \forall A \in D(I)^{Y}$.

(c) $B_{1} \subset B_{2} \Rightarrow f^{-1}\left(B_{1}\right) \subset f^{-1}\left(B_{2}\right)$, where $B_{1}, B_{2} \in$ $D(I)^{Y}$.

(d) $A_{1} \subset A_{2} \Rightarrow f\left(A_{1}\right) \subset f\left(A_{2}\right)$, where $A_{1}, A_{2} \in$ $D(I)^{X}$.

(e) $f\left(f^{-1}(B)\right) \subset B, \forall B \in D(I)^{Y}$.

(f) $A \subset f\left(f^{-1}(A)\right), \forall A \in D(I)^{Y}$.

(g) $(g \circ f)^{-1}(C)=f^{-1}\left(g^{-1}(C)\right), \forall C \in D(I)^{Z}$.

(h) $f^{-1}\left(\bigcup_{\alpha \in \Gamma} B_{\alpha}\right)=\bigcup_{\alpha \in \Gamma} f^{-1} B_{\alpha}$, where $\left\{B_{\alpha}\right\}_{\alpha \in \Gamma} \in$ $D(I)^{Y}$.

(h) $f^{-1}\left(\bigcap_{\alpha \in \Gamma} B_{\alpha}\right)=\bigcap_{\alpha \in \Gamma} f^{-1} B_{\alpha}$, where $\left\{B_{\alpha}\right\}_{\alpha \in \Gamma} \in$ $D(I)^{Y}$.

\section{Interval-valued fuzzy subgroups}

Definition 3.1 $[1,6]$. Let $G$ be a group with the identity $e$ and let $A \in D(I)^{G}$. Then $A$ is called an intervalvalued fuzzy subgroup (in short, $I V G$ ) of $G$ if

(i) $A^{L}(x y) \geq A^{L}(x) \wedge A^{L}(y)$ and $A^{U}(x y) \geq A^{U}(x) \wedge$ $A^{U}(y)$ for any $x, y \in G$.

(ii) $A^{L}\left(x^{-1}\right) \geq A^{L}(x)$ and $A^{U}\left(x^{-1}\right) \geq A^{U}(x)$ for each $x \in G$.

We will denote the set of all IVGs of $G$ as IVG(G).

Result 3.A [1, Proposition 3.1]. Let $G$ be a group with the identity $e$ and let $A \in \operatorname{IVG}(\mathrm{G})$. Then $A\left(x^{-1}\right)=A(x)$ and $A^{L}(x) \leq A^{L}(e), A^{U}(x) \leq A^{U}(e)$ for each $x \in G$.

Result 3.B [6, Proposition 4.6]. If $A \in \operatorname{IVG}(\mathrm{G})$, then $G_{A}=\{x \in G: A(x)=A(e)\}$ is a subgroup of $G$.

Result 3.C [6, Proposition 4.3]. Let $\left\{A_{\alpha}\right\}_{\alpha \in \Gamma} \subset \operatorname{IVG}(\mathrm{G})$. Then $\bigcap_{\alpha \in \Gamma} A_{\alpha} \in \operatorname{IVG}(\mathrm{G})$.

Definition 3.2 [6]. Let $G$ be a group with the identity $e$ and let $A \in \operatorname{IVG}(\mathrm{G})$. Then $A$ is called an intervalvalued fuzzy normal subgroup (in short, IVNG) of $G$ if $A(x y)=A(y x)$ for any $x, y \in G$.
We will denote the set of all IVNGs of $G$ as IVNG(G).

Definition 3.3. Let $A$ be an IVG of a group $G$ and let $\theta: G \rightarrow G$ be a mapping. We define a mapping $A^{\theta}=$ $\left[\left(A^{\theta}\right)^{L},\left(A^{\theta}\right)^{U}\right]: G \rightarrow D(I)$ as follows : For each $g \in G$,

$$
A^{\theta}(g)=A(\theta(g)) \text {. }
$$

For a group $G$, a subgroup $K$ is called a characteristic subgroup if $\theta(K)=K$ for every automorphism $\theta$ of $G$. We now define an analog.

Definition 3.4. Let $A$ be an IVG of a group $G$. Then $A$ is called an interval-valued fuzzy characteristic subgroup of $G$ if $A^{\theta}=A$ for every automorphism $\theta$ of $G$.

Proposition 3.5. Let $G$ be a group, let $A \in D(I)^{G}$ and let $\theta: G \rightarrow G$ be a mapping.

(a) If $A \in \operatorname{IVG}(\mathrm{G})$ and $\theta$ is a homomorphism, then $A^{\theta} \in$ $\operatorname{IVG}(\mathrm{G})$.

(b) If $A$ is an interval-valued fuzzy characteristic subgroup of $G$, then $A \in \mathrm{IVNG}(\mathrm{G})$.

Proof. (a) Let $x, y \in G$. Then

$$
\begin{aligned}
A^{\theta}(x y) & =A(\theta(x y)) \\
& =A(\theta(x) \theta(y)) .[\text { Since } \theta \text { is a homomorphism] }
\end{aligned}
$$

Since $A \in \operatorname{IVG}(\mathrm{G})$,

$$
\begin{aligned}
A^{L}(\theta(x) \theta(y)) & \geq A^{L}(\theta(x)) \wedge A^{L}(\theta(y)) \\
& =\left(A^{\theta}\right)^{L}(x) \wedge\left(A^{\theta}\right)^{L}(y) .
\end{aligned}
$$

Similarly, we have that

$$
A^{U}(\theta(x) \theta(y)) \geq\left(A^{\theta}\right)^{U}(x) \wedge\left(A^{\theta}\right)^{U}(y) .
$$

Thus

$$
\left(A^{\theta}\right)^{L}(x y) \geq\left(A^{\theta}\right)^{L}(x) \wedge\left(A^{\theta}\right)^{L}(y)
$$

and

$$
\left(A^{\theta}\right)^{U}(x y) \geq\left(A^{\theta}\right)^{U}(x) \wedge\left(A^{\theta}\right)^{U}(y) .
$$

On the other hand,

$$
\begin{aligned}
A^{\theta}\left(x^{-1}\right) & =A\left(\theta\left(x^{-1}\right)\right) \\
& =A\left(\theta(x)^{-1}\right)[\text { Since } \theta \text { is a homomorphism }] \\
& =A(\theta(x))[\text { By Result } 3 . A] \\
& =A^{\theta}(x) .
\end{aligned}
$$


Hence $A^{\theta} \in \operatorname{IVG}(\mathrm{G})$.

(b) Let $\theta: G \rightarrow G$ be the automorphism of $G$ defined by $\theta(g)=x^{-1} g x$ for each $g \in G$. Then clearly it is standard result that $\theta$ is an automorphism of $G$, called the inner automorphism induced by $x$. Let $x, y \in G$. Since $A$ is interval-valued fuzzy characteristic, $A^{\theta}=A$. Thus

$$
\begin{aligned}
A(x y) & =A^{\theta}(x y)=A(\theta(x y)) \\
& =A\left(x^{-1}(x y) x\right)[\text { By the definition of } \theta] \\
& =A(y x) .
\end{aligned}
$$

Hence $A \in \operatorname{IVNG}(\mathrm{G})$. This completes the proof.

Remark 3.6. Proposition $3.5(\mathrm{~b})$ is an analog of the result that a characteristic subgroup of a group is normal.

Now we obtain analogs of the concepts of conjugacy, normalizer regarding a group, and their properties.

Definition 3.7. Let $G$ be a group and let $A_{1}, A_{2} \in$ $\operatorname{IVG}(G)$. Then we say that $A_{1}$ is conjugate to $A_{2}$ if there exists an $x \in G$ such that $A_{1}(g)=A_{2}\left(x^{-1} g x\right)$ for each $g \in G$.

It is easy to show that the relation of conjugacy is an equivalence relation on $\operatorname{IVG}(G)$. Hence $\operatorname{IVG}(G)$ is a union of pairwise disjoint classes of interval-valued fuzzy subgroups each consisting of interval-valued fuzzy subgroups which are equivalent to one another. Now we shall obtain an expression giving the number of distinct conjugates of an interval-valued fuzzy subgroups.

Notation. Let $G$ be a group, let $A \in \operatorname{IVG}(\mathrm{G})$ and let $g \in G$. We define a mapping $A^{g}=\left[\left(A^{g}\right)^{L},\left(A^{g}\right)^{U}\right]: G \rightarrow D(I)$ as follows : for each $u \in G, A^{g}(u)=A\left(g^{-1} u g\right)$, i.e., $\left(A^{g}\right)^{L}(u)=A^{L}\left(g^{-1} u g\right)$ and $\left(A^{g}\right)^{U}(u)=A^{U}\left(g^{-1} u g\right)$.

From Proposition 3.5(a), it is clear that $A^{g} \in \operatorname{IVG}(\mathrm{G})$.

Definition 3.8. Let $A$ be an IVG of a group $G$. Then the set $N(A)=\left\{g \in G: A^{g}=A\right\}$ is called the normalizer of $A$.

Proposition 3.9. Let $A$ be an IVG of a group $G$. Then

(a) $N(A)$ is a subgroup of $G$.

(b) $A \in \operatorname{IVNG}(G)$ id and only if $N(A)=G$.

(c) If $G$ is a finite group, then the number of distinct conjugates of $A$ is equal to the index of $N(A)$ in $G$.

Proof. (a) Let $g, h \in N(A)$ and let $u \in G$. Then $A^{g h}(u)=$ $A\left((g h)^{-1} u(g h)\right)=A\left(h^{-1}\left(g^{-1} u g\right) h\right)=A^{h}\left(g^{-1} u g\right)=$ $\left(A^{h}\right)^{g}(u)$. Thus $A^{g h}=\left(A^{g}\right)^{h}=A^{h}=A$. So $g h \in$ $N(A)$. Let $x \in N(A)$ and let $y=x^{-1}$. Let $u \in G$. Then

$$
\begin{aligned}
A^{y}(u) & =A\left(y^{-1} u y\right)=A\left(x u x^{-1}\right)=A\left(\left(x^{-1} u^{-1} x\right)^{-1}\right) \\
& =A\left(x^{-1} u^{-1} x\right)[\text { By Result } 3 . A] \\
& =A^{x}\left(u^{-1}\right)\left[\text { By the definition of } A^{x}\right] \\
& =A\left(u^{-1}\right)\left[\text { Since } A^{x}=A\right] \\
& =A(u) .[\text { By Result } 3 . A]
\end{aligned}
$$

Thus $A^{y}=A$. So $y=x^{-1} \in N(A)$. Hence $N(A)$ is a subgroup of $G$.

(b) $\Rightarrow)$ : Suppose $A \in \operatorname{IVNG}(G)$ and let $g \in G$. Let $u \in G$. Then

$$
\begin{aligned}
A^{g}(u) & =A\left(g^{-1} u g\right)=A\left(\left(g^{-1} u\right) g\right) \\
& =A\left(g\left(g^{-1} u\right)\right)[\text { Since } A \in I V N G(G)] \\
& =A(u) .
\end{aligned}
$$

Thus $A^{g}=A$. So $g \in N(A)$, i.e., $G \subset N(A)$. Hence $N(A)=G$.

$(\Leftarrow)$ : Suppose $N(A)=G$ and let $x, y \in G$. Then

$$
\begin{aligned}
A(x y) & =A\left(x y x x^{-1}\right)=A\left(x(y x) x^{-1}\right) \\
& =A^{x^{-1}}(y x)\left[\text { By the definition of } A^{x^{-1}}\right] \\
& =A(y x) \cdot[\text { By the hypothesis }]
\end{aligned}
$$

Hence $A \in \operatorname{IVNG}(\mathrm{G})$.

(c) Consider the decomposition of $G$ as a union of cosets of $N(A)$,

$$
G=x_{1} N(A) \cup x_{2} N(A) \cup \cdots \cup x_{k} N(A),
$$

where $k$ is the number of distinct cosets, i.e., the index of $N(A)$ in $G$. Let $x \in N(A)$ and choose $i$ such that $1 \leq i \leq$ 
$k$. Let $g \in G$. Then

$$
\begin{aligned}
A^{x_{i} x}(g) & =A\left(\left(x_{i} x\right)^{-1} g\left(x_{i} x\right)\right) \\
& =A\left(x^{-1}\left(x_{i}^{-1} g x_{i}\right) x\right) \\
& =A^{x}\left(x_{i}^{-1} g x_{i}\right) \\
& =A\left(x_{i}^{-1} g x_{i}\right)[\text { Since } x \in N(A)] \\
& =A^{x_{i}}(g) .
\end{aligned}
$$

Thus $A^{x_{i} x}=A^{x_{i}}$ for each $x \in N(A)$ and $1 \leq i \leq k$. So any two elements of $G$ which lie in the same coset $x_{i} N(A)$ give rise to the same conjugate $A^{x_{i}}$ of $A$. Now we show that two distinct cosets give two distinct conjugates of $A$. Assume that $A^{x_{i}}=A^{x_{j}}$, where $i \neq j$ and $1 \leq i \leq k$, $1 \leq j \leq k$. Let $g \in G$. Then

$$
A^{x_{i}}(g)=A^{x_{j}}(g) \text {, i.e., } A\left(x_{i}^{-1} g x_{i}\right)=A\left(x_{j}^{-1} g x_{j}\right) .
$$

Let $h \in G$ such that $g=x_{j} h x_{j}^{-1}$. Then, by (3.2),

$$
\begin{aligned}
& A\left(x_{i}^{-1} x_{j} h x_{j}^{-1} x_{i}\right)=A\left(x_{j}^{-1} x_{j} h x_{j}^{-1} x_{j}\right) \\
\Rightarrow & A\left(\left(x_{i}^{-1} x_{j}\right) h\left(x_{j}^{-1} x_{i}\right)\right)=A(h), \\
& \text { i.e., } A\left(\left(x_{j}^{-1} x_{i}\right)^{-1} h\left(x_{j}^{-1} x_{i}\right)\right)=A(h) \\
\Rightarrow & A^{x_{j}^{-1} x_{i}}(h)=A(h), \text { i.e., } A^{x_{j}^{-1} x_{i}}=A .
\end{aligned}
$$

Thus $x_{j}^{-1} x_{i} \in N(A)$. So $x_{i} N(A)=x_{j} N(A)$. Since (3.1) represent a partition of $G$ into pairwise disjoint cosets and $i \neq j$, this is not possible. Hence the number of distinct conjugates of $A$ is equal to the index of $N(A)$ in $G$. This completes the proof.

Remark 3.10. Proposition 3.9(b) illustrates the motivation behind the term "normalizer" and it shows the analogy with the fact that a subgroup $H$ of a group $G$ is normal in $G$ if and only if the normalizer of $H$ in $G$ is equal to $G$ itself. And Proposition 3.9(c) is an analog of a basic result in group theory.

Definition 3.11 [4]. Let $A$ be an IVG of a group $G$ and let $x \in G$. We define two mappings $A x=\left[A x^{L}, A x^{U}\right]: G \rightarrow D(I)$ and $x A=\left[x A^{L}, x A^{U}\right]: G \rightarrow D(I)$ as follows, respectively: For each $g \in G, A x(g)=A\left(g x^{-1}\right)$ and $x A(g)=$ $A\left(x^{-1} g\right)$. Then $A x[\operatorname{resp} . x A]$ is called the intervalvalued fuzzy right [resp.left] coset of $G$ determined by $x$ and $A$.

Lemma 3.12. Let $A$ be an IVG of a group $G$ and let $K=$ $\{x \in G: A x=A e\}$,

where $e$ denotes the identity element of $G$. Then $K$ is a subgroup of $G$. Furthermore, $G_{A}=K$.

Proof. Let $k \in K$ and let $g \in G$. Then $A k(g)=A e(g)$. Thus $A\left(g k^{-1}\right)=A(g)$. In particular, $A\left(e k^{-1}\right)=A(e)$, i.e., $A\left(k^{-1}\right)=A(e)$. Thus $k^{-1} \in G_{A}$. By Result 3.B, $G_{A}$ is a subgroup of $G$. Thus $k \in G_{A}$. So $K \subset G_{A}$. Now let $h \in G_{A}$. Then

$$
A(h)=A(e) .
$$

Let $g \in G$. Then $A h(g)=A\left(g h^{-1}\right)$ and $A e(g)=A(g)$. Thus

$$
\begin{aligned}
A^{L}\left(g h^{-1}\right) & \geq A^{L}(g) \wedge A^{L}\left(h^{-1}\right) \\
& =A^{L}(g) \wedge A^{L}(h)[\text { By Result } 3 . A] \\
& =A^{L}(g) \wedge A^{L}(e)[\text { By }(3.3)] \\
& =A^{L}(g) .[\text { By Result } 3 . A]
\end{aligned}
$$

Similarly, we have that $A^{U}\left(g h^{-1}\right) \geq A^{U}(g)$. Also,

$$
\begin{aligned}
A^{L}(g) & =A^{L}\left(g h^{-1} h\right) \geq A^{L}\left(g h^{-1}\right) \wedge A^{L}(h) \\
& =A^{L}\left(g h^{-1}\right) \wedge A^{L}(e)[\text { By }(3.3)] \\
& =A^{L}\left(g h^{-1}\right) .[\text { By Result } 3 . A]
\end{aligned}
$$

Similarly, we have that $A^{U}(g) \geq A^{U}\left(g h^{-1}\right)$. So $A\left(g h^{-1}\right)=A(g)$, i.e., $A h=A e$, i.e., $h \in K$. Hence $G_{A} \subset K$. Therefore $G_{A}=K$. This completes the proof.

Corollary 3.12 [6, Proposition 5.4]. Let $G$ be a group. If $A \in \operatorname{IVNG}(G)$, then $G_{A} \triangleleft G$.

Proof. Let $g \in G$ and let $x \in G_{A}$. Then

$$
\begin{aligned}
A\left(g^{-1} x g\right) & =A\left(g g^{-1} x\right)[\text { Since } A \in \operatorname{IVNG}(G)] \\
& =A(x) \\
& =A(e) .\left[\text { Since } x \in G_{A}\right]
\end{aligned}
$$

Thus $g^{-1} x g \in G_{A}$. Hence $G_{A} \triangleleft G$. 
For a group $G$, the commutator $[x, y]$ of two elements $x, y$ in $G$ is defined as $[x, y]=x^{-1} y^{-1} x y$. If $x y=y x$, then obviously $[x, y]=e$. Thus $G$ is abelian if $[x, y]=e$ for all $x, y \in G$. This motivates the following definition.

Remark 3.13. A special case of Lemma 3.12 is implicit in Theorem 2.12 in [4], where it was tacitly assumed that $A$ is interval-valued fuzzy normal. But, as we see now, it is not necessarily to assume that $A$ is interval-valued fuzzy normal, and this fact straightens the proof of the interval-valued fuzzy Lagrange's theorem [4, Theorem 4.12].

Definition 3.14. Let $A$ be an IVG of a group $G$. Then $A$ is said to be interval-valued fuzzy abelian if $A([x, y])=A(e)$ for any $x, y \in G$.

Result 3.D [4, Theorem 2.12]. Let $A \in \operatorname{IVG}(\mathrm{G})$. Then $A \in \operatorname{IVNG}(\mathrm{G})$ if and only if $A^{L}([x, y]) \geq A^{L}(x)$ and $A^{U}([x, y]) \geq A^{U}(x)$ for any $x, y \in G$.

Analogous to some well-known properties of abelian group, we prove.

Theorem 3.15. (a) An interval-valued fuzzy abelian subgroup of a group is interval-valued fuzzy normal.

(b) Given an interval-valued fuzzy abelian subgroup of $G$, there is a normal subgroup $N$ of $G$ such that $G / N$ is abelian.

Proof. (a) Let $A$ be an interval-valued fuzzy abelian subgroup of $G$. Let $x, y \in G$. Then, by Result 3.A, $A^{L}([x, y])=A^{L}(e) \geq A^{L}(x)$ and $A^{U}([x, y])=A^{U}(e) \geq$ $A^{U}(x)$. Hence, by Result 3.D, $A \in \operatorname{IVNG}(\mathrm{G})$.

(b) Let $A$ be an interval-valued fuzzy abelian subgroup

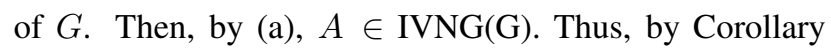
3.12, $G_{A} \triangleleft G$. Also, it is easy to see that $G^{\prime} \subset G_{A}$, where $G^{\prime}$ denotes the commutator subgroup of $G$ (i.e., the subgroup generated by all elements $[x, y], x, y \in G)$. Hence $G / G_{A}$ is abelian.
The following is the immediate result of Definition 3.2 and Result 3.C.

Proposition 3.16. If $\left\{A_{\alpha}\right\}_{\alpha \in \Gamma}$ is a family of IVNGs of a group $G$, then $\bigcap_{\alpha \in \Gamma} A_{\alpha} \in \operatorname{IVNG}(\mathrm{G})$. Furthermore, if $A, B \in \mathrm{IVNG}(\mathrm{G})$, then $A \cap B \in \mathrm{IVNG}(\mathrm{G})$.

It is a standard result in group theory that if $G$ is a group, $H \leq G, K \leq G$ and $H \triangleleft G$, then $H \cap K \triangleleft K$ is normal in $K$. Now we derive an analog for interval-valued fuzzy subgroups.

Proposition 3.17. Let $G$ be a group and let $A \in \operatorname{IVG}(\mathrm{G})$, $B \in \operatorname{IVNG}(\mathrm{G})$. Then $A \cap B$ is an interval-valued fuzzy normal subgroup of the group $G_{A}$.

Proof. It is clear that $G_{A}$ is a subgroup of $G$ by Result 3.B. By Proposition 3.16, $A \cap B \in \mathrm{IVG}(\mathrm{G})$. Thus $A \cap B \in$ $\operatorname{IVG}\left(G_{A}\right)$. Let $x, y \in G_{A}$. Since $G_{A}$ is a subgroup of $G$, $x y \in G_{A}$ and $y x \in G_{A}$. Thus $A(x y)=A(y x)=A(e)$. Since $B \in \operatorname{IVNG}(\mathrm{G}), B(x y)=B(y x)$. So

$$
\begin{aligned}
(A \cap B)(x y) & =\left[A^{L}(x y) \wedge B^{L}(x y), A^{U}(x y) \wedge B^{U}(x y)\right] \\
& =\left[A^{L}(y x) \wedge B^{L}(y x), A^{U}(y x) \wedge B^{U}(y x)\right] \\
& =(A \cap B)(y x) .
\end{aligned}
$$

Hence $A \cap B \in \operatorname{IVNG}\left(G_{A}\right)$.

\section{Interval-valued fuzzy cosets}

Result 4.A [4, Theorem 2.9]. Let $A$ be an IVG of a group $G$. Then the followings are equivalent :

(a) $A^{L}\left(x y x^{-1}\right) \geq A^{L}(y)$ and $A^{U}\left(x y x^{-1}\right) \geq A^{U}(y)$ for any $x, y \in G$.

(b) $A\left(x y x^{-1}\right)=A(y)$ for any $x, y \in G$.

(c) $A \in \operatorname{IVNG}(\mathrm{G})$.

(d) $x A=A x$ for each $x \in G$.

(e) $x A x^{-1}=A$ for each $x \in G$.

Remark 4.1. We shall restrict ourselves in the subsequent discussion, without any loss of generality, with 
interval-valued fuzzy right cosets only(corresponding results for interval-valued fuzzy left cosets could be obtained without any difficulty). Consequently from now on we call an interval-valued fuzzy right coset an interval-valued fuzzy coset and denote it as $A x$ for each $x \in G$.

Definition 4.2 [4]. Let $A$ be an IVG of a finite group $G$. Then the cardinality $|G / A|$ of $G / A$ is called an index of $A$, where $G / A$ denotes the set of all interval-valued fuzzy cosets of $A$.

Result 4.B [4, Proposition 3.7]. Let $A$ be an IVNG of a group $G$. We define an operation $*$ on $G / A$ as follows : For any $x, y \in G, A x * A y=A x y$. Then $(G / A, *)$ is a group. In this case, $G / A$ is called the interval-valued fuzzy quotient group by $A$.

Result 4.C [4, Theorem 4.12]. Let $A$ be an IVG of a finite group $G$. Then the index of $A$ divides the order of $G$.

It is a well-known result in group theory that subgroup of index 2 is a normal subgroup. We now obtain an analog of a generalization of this result.

Proposition 4.3. Let $A$ be an IVG of a finite group $G$ such that the index of $A$ is $p$, where $p$ is the smallest prime

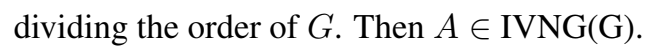

Proof. By Result 3.B, $G_{A}$ is a subgroup of $G$. Since $A$ is an IVG of $G$ such that the index of $A$ is $p$, by Lemma 3.12 and Result 4.C, $G_{A}$ has index $p$ in $G$, i.e., $G_{A}$ has $p$ distinct (right) cosets, say, $\left\{G_{A} x_{i}: 1 \leq i \leq p\right\}$. Now consider the permutation representation of $G$ on the cosets of $G_{A}$ given by the map $\pi: x \rightarrow \pi_{x^{-1}}$, where $\pi_{x^{-1}}: G_{A} x_{i} \rightarrow G_{A} x_{i} x^{-1}, 1 \leq i \leq p$. Since the index of $G_{A}$ in $G$ is $p, \pi$ is an isomorphism of $G$ into the symmetric group $S_{p}$. Furthermore, $\operatorname{Ker} \pi=\operatorname{Core}\left(G_{A}\right)$, where $\operatorname{Core}\left(G_{A}\right)$ denotes the intersection of all the conjugates $g^{-1} G_{A} g, g \in G$. By the fundamental theorem of homomorphisms of groups and using Lagrange's theorem, the order of $G / \operatorname{Core}\left(G_{A}\right)$ divides $p$ ! which is the order of
$S_{p}$. Furthermore,

$$
G / \operatorname{Core}\left(G_{A}\right) \cong\left(G / G_{A}\right)\left(G_{A} / \operatorname{Core}\left(G_{A}\right)\right)
$$

and the order of $G / G_{A}$ is $p$. Thus it follows that the order of $G_{A} / \operatorname{Core}\left(G_{A}\right)$ divides $(p-1)$ !. Since the order of $G_{A}$ divides the order of $G, G_{A}=\operatorname{Core}\left(G_{A}\right)$; otherwise we get a contradiction to the fact that $p$ is the smallest prime dividing the order of $G$. Since $\operatorname{Core}\left(G_{A}\right)$ is a normal subgroup of $G, G_{A}$ is a normal subgroup of $G$. Now consider the quotient group $G / H$. Since the order of $G / G_{A}$ is $p, G / G_{A}$ is abelian. Let $x, y \in G$. Then $\left(G_{A} x\right)\left(G_{A} y\right)=\left(G_{A} y\right)\left(G_{A} x\right)$. Thus $G_{A} x y=G_{A} y x$. So there exists an $h \in G_{A}$ such that $x y=h y x$. Then

$$
\begin{aligned}
A^{L}(x y) & =A^{L}(h y x) \geq A^{L}(h) \wedge A^{L}(y x) \\
& =A^{L}(e) \wedge A^{L}(y x)=A^{L}(y x) .
\end{aligned}
$$

Similarly, we have that $A^{U}(x y) \geq A^{U}(y x)$. Also, we have that $A^{L}(y x) \geq A^{L}(x y)$ and $A^{U}(y x) \geq A^{U}(x y)$. So $A(x y)=A(y x)$ for any $x, y \in G$. Hence $A \in \operatorname{IVNG}(\mathrm{G})$. This completes the proof.

The following is the immediate result of Proposition 4.3 .

Corollary 4.3. Let $A$ be an IVG of a group $G$ such that the

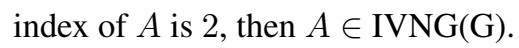

It is well-known in group theory that $\theta$ is a homomorphism of a group $G$ into itself whose kernel is $N$, then $\theta$ induces a homomorphism from $G / N$ into itself. Now we derive an analog of the following result.

Proposition 4.4. Let $A$ be an IVNG of a group $G$ and let $\theta$ be an homomorphism of $G$ into itself such that $\theta\left(G_{A}\right)=G_{A}$. Then $\theta$ induces a homomorphism $\bar{\theta}$ of the interval-valued fuzzy cosets of $A$ defined as follows : $\bar{\theta}(A x)=A \theta(x)$ for each $x \in G$.

Proof. Suppose $x, y \in G$ such that $A x=A y$. Then $A x(x)=A y(x)$ and $A x(y)=A y(y)$. Thus $A(e)=$ $A\left(x y^{-1}\right)=A\left(y x^{-1}\right)$. So $x y^{-1}, y x^{-1} \in G_{A}$. Since 


$$
\begin{array}{r}
\theta\left(G_{A}\right)=G_{A}, \theta\left(x y^{-1}\right), \theta\left(y x^{-1}\right) \in G_{A} . \text { Then } \\
A\left(\theta\left(x y^{-1}\right)\right)=A\left(\theta\left(y x^{-1}\right)\right)=A(e) .
\end{array}
$$

Let $g \in G$. Then

$$
\begin{aligned}
(A \theta(x))^{L}(g)= & A^{L}\left(g \theta(x)^{-1}\right) \\
= & A^{L}\left(g \theta\left(x^{-1}\right)\right)[\text { Since } \theta \text { is a homomo } \\
= & A^{L}\left(g \theta\left(y^{-1}\right) \theta(y) \theta\left(x^{-1}\right)\right) \\
\geq & A^{L}\left(g \theta\left(y^{-1}\right)\right) \wedge A^{L}\left(\theta(y) \theta\left(x^{-1}\right)\right) \\
& {[\text { Since } A \in \operatorname{IVG}(\mathrm{G})] } \\
= & A^{L}\left(g \theta\left(y^{-1}\right)\right) \wedge A^{L}\left(\theta\left(y x^{-1}\right)\right) \\
& {[\operatorname{Since} \theta \text { is a homomorphism }] } \\
= & (A \theta(y))^{L}(g) \wedge A^{L}(e)[\text { By }(4.1)] \\
= & (A \theta(y))^{L}(g) .[\text { By Result 3.A] }
\end{aligned}
$$$$
=A^{L}\left(g \theta\left(x^{-1}\right)\right)[\text { Since } \theta \text { is a homomorphism }]
$$

Similarly, we have that $(A \theta(x))^{U}(g) \geq(A \theta(y))^{U}(g)$. Also, we have that $(A \theta(y))^{L}(g) \geq(A \theta(x))^{L}(g)$ and $(A \theta(y))^{U}(g) \geq(A \theta(x))^{U}(g)$. Thus $A \theta(x)=A \theta(y)$. So $\bar{\theta}$ is well-defined. Now let $x, y \in G$. Then

$$
\begin{aligned}
\bar{\theta}(A x * A y) & =\bar{\theta}(A x y)[\text { By Result } 4 . B] \\
& =A \theta(x y)[\text { By the definition of } \bar{\theta}] \\
& =A \theta(x) \theta(y)[\text { Since } \theta \text { is a homomorphism }] \\
& =A \theta(x) * A \theta(y)[\text { By Result } 4 . B] \\
& =\bar{\theta}(A x) * \bar{\theta}(A y) .[\text { By the definition of } \bar{\theta}]
\end{aligned}
$$

Hence $\bar{\theta}$ is a homomorphism. This completes the proof.

Corollary 4.4-1. In the same hypothesis as in Proposition 4.4, if $\theta$ is an automorphism and $G$ is finite, then $\bar{\theta}$ is an automorphism.

Proof. Since $G$ has finite order, it is easy to see that $\theta$ has finite order. Suppose that $\theta$ has order $k$. Then $\theta^{k}=i d_{G}$, where $i d_{G}$ denotes the identity mapping. Let $x, y \in G$ such that $\bar{\theta}(A x)=\bar{\theta}(A y)$. Then, by the definition of $\bar{\theta}$, $A \theta(x)=A \theta(y)$.

Since $\theta^{k}=i d_{G}, \theta^{k}(x)=x$ and $\theta^{k}(y)=y$. Thus $A x=$ $A \theta^{k}(x)=A \theta^{k}(y)=A y$.

So $\bar{\theta}$ is injective. Hence $\bar{\theta}$ is an automorphism.
Corollary 4.4-2. In the same hypothesis as in Proposition 4.4 , if $\bar{\theta}$ is an automorphism and $G_{A}=(e)$, then $\theta$ is an automorphism.

Proof. Let $x, y \in G$ such that $\theta(x)=\theta(y)$. Then $A \theta(x)=A \theta(y)$, i.e., $\bar{\theta}(A x)=\bar{\theta}(A y)$. Since $\bar{\theta}$ is injective, $A x=A y$. Then $A x(y)=A y(y)$. Thus $A\left(y x^{-1}\right)=A(e)$. So $y x^{-1} \in G_{A}$. Since $G_{A}=(e), y x^{-1}=e$. Thus $x=y$. So $\theta$ is injective. Hence $\theta$ is an automorphism.

The motivation of the following result stems from the standard theorem in group theory that if $\theta$ is an automorphism of $G$ and $N$ is a normal subgroup of $G$ such that $N^{\theta} \subset N$, then $\theta$ induces an automorphism of the quotient group $G / N$ into itself.

Remark 4.5. In Proposition 4.4, we have assumed $A$ to be interval-valued fuzzy normal instead of assuming only that $A$ is an interval-valued fuzzy subgroup. This has been done to ensure that the law of composition of interval-valued fuzzy cosets is well-defined, and this fact is used in the proof of Proposition 4.4 to show that $\bar{\theta}$ is a homomorphism(refer to Result 4.B). However, it is clear from the proof that to show $\bar{\theta}$ is well-defined it is not necessary to assume $A$ to be interval-valued fuzzy normal.

Proposition 4.6. Let $A$ be an IVNG of a group $G$ and let $\theta$ be an automorphism of $G$ such that $A^{\theta}=A$ (recall the definition of $A^{\theta}$ given by Definition 3.3). Then $\theta$ induces an automorphism $\bar{\theta}$ of $G / A$ defined as follows : for each $x \in G, \bar{\theta}(A x)=A \theta(x)$.

Proof. Let $x, y \in G$ such that $A x=A y$. We show that $\bar{\theta}(A x)=\bar{\theta}(A y)$, i.e., $A \theta(x)(g)=A \theta(y)(g)$ for each $g \in G$. Let $g \in G$. Since $\theta$ is an automorphism of $G$, there exists a $g^{*} \in G$ such that $\theta\left(g^{*}\right)=g$. Since $A x=A y$, $A x\left(g^{*}\right)=A y\left(g^{*}\right)$, i.e., $A\left(g^{*} x^{-1}\right)=A\left(g^{*} y^{-1}\right)$. Since $A^{\theta}=A, A^{\theta}\left(g^{*} x^{-1}\right)=A^{\theta}\left(g^{*} y^{-1}\right)$. By Definition 3.3, $A\left(\theta\left(g^{*} x^{-1}\right)\right)=A\left(\theta\left(g^{*} y^{-1}\right)\right)$. Since $\theta$ is an automorphism of $G, A\left(\theta\left(g^{*}\right) \theta\left(x^{-1}\right)\right)=A\left(\theta\left(g^{*}\right) \theta\left(y^{-1}\right)\right)$. Thus $A\left(g \theta\left(x^{-1}\right)\right)=A\left(g \theta\left(y^{-1}\right)\right)$, i.e., $A \theta(x)(g)=A \theta(y)(g)$. So $\bar{\theta}(A x)=\bar{\theta}(A y)$. Hence $\bar{\theta}$ is well-defined. The proof 
of the fact that $\bar{\theta}$ is a homomorphism is analogous to the corresponding part of the proof of Proposition 4.4, and thus we omit the details. Now suppose $A x \in \operatorname{Ker} \bar{\theta}$ for each $x \in G$. Then $\bar{\theta}(A x)=A \theta(x)=A e$. Let $g \in G$. Then $A \theta(x)(\theta(g))=A e \theta(g)$, i.e., $A\left(\theta(g) \theta\left(x^{-1}\right)\right)=A \theta(g)$. Thus $A \theta\left(g x^{-1}\right)=A \theta(g)$, i.e., $A^{\theta}\left(g x^{-1}\right)=A^{\theta}(g)$. Since $A^{\theta}=A, A\left(g x^{-1}\right)=A(g)$. Then $A x(g)=A e(g)$. Thus $A x=A e$, i.e., $\operatorname{Ker} \bar{\theta}=\{A e\}$. So $\bar{\theta}$ is injective. Hence $\bar{\theta}$ is an automorphism of $G / A$. This completes the proof.

Theorem 4.7. Let $A$ be an IVG of a finite group $G$ and let $x, y \in G$. Then $G_{A} x=G_{A} y$ if and only if $A x=A y$.

Proof. By Result 3.B and Lemma 3.12, $G_{A}$ is a subgroup of $G$ and $G_{A}=\{x \in G: A x=A e\}$.

$(\Rightarrow)$ : Suppose $G_{A} x=G_{A} y$ for any $x, y \in G$. Then $x y^{-1} \in G_{A}$. Thus $A x y^{-1}=A e$. Let $g \in G$. Then $A x y^{-1}(g)=A e(g)$, i.e., $A\left(g y x^{-1}\right)=A(g)$. Replacing $g$ by $g y^{-1}$, which is also an arbitrary element of $G$, we get that $A\left(g x^{-1}\right)=A\left(g y^{-1}\right)$ for each $y \in G$. Thus $A x(g)=$ $A y(g)$ for each $y \in G$. So $A x=A y$.

$(\Leftarrow)$ : Suppose $A x=A y$ for any $x, y \in G$ and let $g \in G$. Then $A x(g)=A y(g)$, i.e., $A\left(g x^{-1}\right)=A\left(g y^{-1}\right)$. In particular, $A\left(y x^{-1}\right)=A\left(y y^{-1}\right)=A(e)$. Thus $y x^{-1} \in G_{A}$. So $G_{A} x=G_{A} y$. This completes the proof.

Remark 4.8. Proposition 4.6 shows that there is a oneto-one correspondence between the (right) cosets of $G_{A}$ in $G$ and the interval-valued fuzzy cosets of $A$, given by the mapping $x \leftrightarrow A x$ for each $x \in G$. Hence we see that the subgroup $G_{A}$ plays a key role in the analysis of intervalvalued fuzzy cosets.

\section{References}

[1] R. Biswas, "Rosenfeld's fuzzy subgroups with interval-valued membership functions," Fuzzy set and systems, vol. 63, pp. 87-90, 1995.

[2] M. S. Cheong and K. Hur, "Interval-valued fuzzy generalized bi-ideals of a semigroup," International
J.Fuzzy Logic and Intelligent Systems, vol. 11, pp. 259-266, 2011.

[3] J. Y. Choi, S. K. Kim and K. Hur, "Interval-valued smooth topological spaces," Honam Math.J., vol. 32, pp. 711-738, 2010.

[4] S. Y. Jang, K. Hur and P. K. Lim, "Interval-valued fuzzy normal subgroups," International J.Fuzzy Logic and Intelligent Systems, vol. 12, pp. 205-214, 2012.

[5] H. W. Kang, "Interval-valued fuzzy subgroups and homomorphisms," Honam Math.J., vol.33, 2011.

[6] H. W. Kang and K. Hur, "Interval-valued fuzzy subgroups and subrings," Honam Math.J., vol. 32, pp. 543-617, 2010.

[7] K. C. Lee, H. W. Kang and K. Hur, "Interval-valued fuzzy generalited bi-ideals of a semigroup," Honam Math.J., vol. 33, 2011.

[8] T. K. Mondal and S. K. Samanta, "Topology of interval-valued fuzzy sets," Indian J.Pure Appl.Math., vol. 30, pp. 20-38, 1999.

[9] L. A. Zadeh, "Fuzzy sets," Inform. and Control, vol. 8, 1965. 338-353.

[10] L. A. Zadeh, "The concept of a linguistic variable and its application to approximate reasoning I," Inform. Sci., vol. 8, pp. 199-249, 1975.

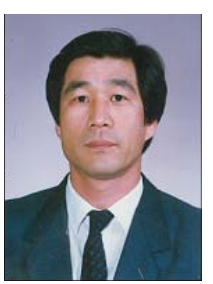

\section{Keon Chang Lee}

Professor in Dongshin University

His research interests are Category Theory, Hyperspace and Topology.

Phone : 061-330-3313

E-mail : kclee@dsu.ac.kr 


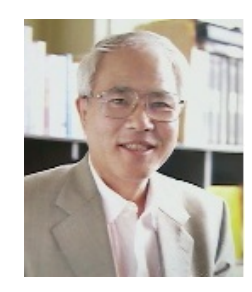

Kul Hur

Professor in Wonkwang University His research interests are Category Theory, Hyperspace and Topology. Phone : 063-850-6081

E-mail : kulhur@wonkwang.ac.kr

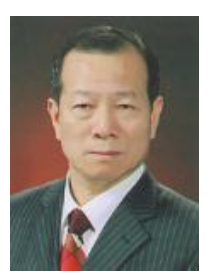

\section{Pyung Ki Lim}

Professor in Wonkwang University

His research interests are Category

Theory, Hyperspace and Topology.

Phone : 063-850-6559

E-mail : pklim@wonkwang.ac.kr 